\title{
Bleeding events in COVID-19: the other side of the coin?
}

\author{
Antonietta Coppola, Anna Annunziata, Maria Rosaria Gioia, Giuseppe Fiorentino \\ Department of Respiratory Pathophysiology, Monaldi-Cotugno Hospital, Naples, Italy
}

\begin{abstract}
We present three cases of patients affected by severe SARSCoV-2-related pneumonia treated with a low molecular weight heparin for prevention or treatment of pulmonary embolism, who presented a major bleed, in particular an ileopsoas haematoma that caused severe anaemia; in one case it was fatal. In the recent outbreak of novel coronavirus infection, significantly abnormal coagulation parameters in SARS-CoV-2 infection occur very often, but complications in the opposite direction such as bleeding diathesis are very rare. In these cases, there are different levels of gravity: for one patient the major bleed required the anticoagulant therapy to be stopped until bleeding stabilized, one patient needed interventional radiology and one patient died.
\end{abstract}

\section{Introduction}

Severe acute respiratory syndrome coronavirus 2 (SARS-CoV2) is a betacoronavirus that belongs to the family Coronaviridae and the order Nidovirales [1]. On December 2019, a series of pneumonia cases caused by a SARS-CoV-2 outbreak was identified in Wuhan, Hubei, China and rapidly spread across the world.

Correspondence: Antonietta Coppola, Ospedale Monaldi, via L. Bianchi, 80131 Napoli, Italy.

Tel. +39.81 .7067333 .

E-mail: antonietta.coppola84@gmail.com

Contributions: All the authors made a substantive intellectual contribution. All the authors have read and approved the final version of the manuscript and agreed to be accountable for all aspects of the work.

Conflict of interest: The authors declare that they have no competing interests, and all authors confirm accuracy.

Key words: COVID-19; LMWH; ileopsoas bleeding.

Received for publication: 15 December 2020.

Accepted for publication: 28 February 2021.

${ }^{\text {CC }}$ Copyright: the Author(s), 2021

Licensee PAGEPress, Italy

Monaldi Archives for Chest Disease 2021; 91:1739

doi: 10.4081/monaldi.2021.1739

This article is distributed under the terms of the Creative Commons Attribution Noncommercial License (by-nc 4.0) which permits any noncommercial use, distribution, and reproduction in any medium, provided the original author(s) and source are credited.
This virus mainly creates a severe acute respiratory syndrome (COVID-19), with fever, cough, asthenia, interstitial pneumonia and, eventually, acute respiratory distress syndrome. Patient management mainly focuses on supportive care: oxygenation, fluid management and treatments with multiple drugs such as antiviral therapies, antibiotics, steroids, nonsteroidal anti- inflammatory drugs and immunosuppressive drugs. Many patients require noninvasive ventilation (NIV) support or C-PAP (Continuous Positive Airway Pressure) whereas others need invasive ventilation. In the available studies, COVID-19 patients showed alterations to coagulation tests, with a significant increase of D-dimer levels associated with severity of illness and adverse outcomes [2]. Besides that, a high risk for venous thromboembolism has been highlighted, with high prevalence in patients of symptomatic acute pulmonary embolism and deep vein thrombosis [3]. Therefore, currently, low molecular weight heparin (LMWH) has become part of the clinical management of hospitalized COVID19 patients, even though evidence about the right prophylactic dose is still lacking. In this scenario, we present three cases of major abdominal bleeding occurring in patients with bilateral interstitial pneumonia and a positive SARS-CoV-2 throat swab, supported with C-PAP ventilation.

The first patient is a 71-year-old man, affected by bilateral interstitial pneumonia complicated by pulmonary embolism and a positive SARS-CoV-2 nasopharyngeal swab. The comorbidities were arterial hypertension, chronic ischaemic heart disease and pulmonary emphysema. His body weight was $62 \mathrm{~kg}$ at admission. He was supported with C-PAP, with $10 \mathrm{~cm} \mathrm{H}_{2} \mathrm{O}$ positive end expiratory pressure (PEEP) and 55\% fraction inspired oxygen $\left(\mathrm{FiO}_{2}\right)$. $\mathrm{He}$ was treated with antiviral drugs, in particular $100 \mathrm{mg} /$ day remdesivir, $40 \mathrm{mg}$ methylprednisolone twice daily and $6000 \mathrm{UI}$ LMWH as enoxaparin once daily. We used a dosage of enoxaparin adjusted for body weight and inlight of acute renal failure (creatinine $2,1 \mathrm{mg} / \mathrm{dl}$, glomerular filtration rate $34 \mathrm{ml} / \mathrm{min}$ ) and monitoring the anticoagulant effect using the anti-Factor Xa activity as suggested by hematologist consultant. Suddenly, 10 days after admission to our sub-intensive unit, he started complaining of severe abdominal pain. Blood pressure decreased to $80 / 60 \mathrm{mmHg}$ and the blood test showed haemoglobin $(\mathrm{Hb})$ of 8.8 $\mathrm{g} / \mathrm{dl}$ (from $13.7 \mathrm{~g} / \mathrm{dl} 2$ days before); prothrombin time (PT) was normal, platelet (PTL) count was $164 \times 10^{3} / \mathrm{mL}$ and D-Dimer level was $781 \mathrm{ng} / \mathrm{ml}$. The abdominal CT scan showed a large pelvic blood collection anterior to the left ileopsoas muscle. The lesion showed enhanced contrast tardive above the ischiopubic branch, a sign of active venous bleeding. According to the vascular surgeon, it was decided to opt for clinical observation (transfusions and subsequent blood count associated with elastocompressive dressing). Inflammation indices and haemoglobin values normalized in a few days. We observed reabsorption of the haematoma in 10 days.

The second case regarded an 82-year-old man, supported with C-PAP with $12 \mathrm{~cm} \mathrm{H}_{2} \mathrm{O}$ PEEP and $60 \% \mathrm{FiO}_{2}$. The comorbidities 
were arterial hypertension, chronic ischaemic heart disease and Alzheimer's disease. His body weight was $67 \mathrm{~kg}$. Renal function was conserved (creatinine $1 \mathrm{mg} / \mathrm{dl}$, VFG: $76 \mathrm{ml} / \mathrm{min}$ ). He was treated with azithromycin, steroids and 6000 UI enoxaparina once daily. We used a prophylactic weight-adjusted dosage of enoxaparin (10UI/Kg once daily). Suddenly, 14 days after admission to the hospital, he started complaining of severe abdominal pain; blood pressure decreased to $80 / 60 \mathrm{mmHg}$ and the blood test showed $\mathrm{Hb}$ of $7.1 \mathrm{~g} / \mathrm{dl}$ (from $13.1 \mathrm{~g} / \mathrm{dl}$ ), and D-dimer level of 980 $\mathrm{ng} / \mathrm{ml}$. The abdominal CT scan demonstrated a large $(180 \mathrm{~mm})$ pelvic blood collection in the right iliac fossa, indissociable from the ileopsoas muscle (Figure 1). The patient received radiological embolization of the left epigastric inferior artery. In the following days, the bleeding stopped, confirmed by imaging; haemodynamic balance improved, and haemoglobin and inflammation index values normalized.

The last case is about a 61-year-old man, affected by arterial hypertension and glaucoma, who was admitted to the semi-intensive respiratory unit for acute respiratory failure related to COVID-19 interstitial pneumonia. His body weight was $61 \mathrm{~kg}$. At the beginning, he was supported with high-flow nasal cannula oxygen and treated with methylprednisolone, piperacillin/tazobactam, 6000 UI enoxaparin once daily and vitamin C, but for the worsening of gas exchanges he was supported by CPAP. We used a prophylactic dosage of LMWH adjusted for weight, because there were no clinical signs of VTE and no documented thrombotic manifestation. Suddenly, 17 days after admission, similarly to the case previously described, the patient complained of severe abdominal pain, with clinical signs of haemorrhagic shock. The CT scan revealed a large $(93 \times 90 \mathrm{~mm})$ blood collection in the left ileopsoas muscle with multiple blushes as active arterial bleeding associated with haemoperitoneum (Figure 2). Haemoglobin was $7.1 \mathrm{~g} / \mathrm{dl}$ (from $14 \mathrm{~g} / \mathrm{dl}$ ). PTL, prothrombin time (PT) and partial thromboplastin time (PTT) were normal and D-dimer was $1081 \mathrm{ng} / \mathrm{ml}$ but the haemoglobin value rapidly decreased to $3.3 \mathrm{~g} / \mathrm{dl}$. The patient was transported to the operating room but he died during preparation for surgery.

\section{Discussion}

Iliopsoas haematoma is a distinct clinical entity that can present as a rare life-threatening event and may develop in association with anticoagulation states, coagulopathies and haemodialysis, or anticoagulant/antithrombotic therapy dosage [4]. Genesis of the bleeding in patients affected by COVID-19 is not clear.

Anticoagulation therapy is a risk for bleeding and in these cases the three patients has been treated with LMWH at weightadjusted dosage, that usually represents a low bleeding risk, and we probably have to consider different factors. One patient had acute renal failure with a pulmonary embolism documented, for the other two patients the renal function was conserved and there were no signs of thrombotic manifestation or documented VTE. Moreover, they were not critically ill patients.

Firstly, patients were supported with C-PAP, which certainly gives a positive pression in the airway and consequentially increases intrabdominal pressure. This could be a factor that favours bleeding.

Secondly, in COVID-19, cough is a common symptom and could have led to a relevant increase in abdominal pressure, causing venous rupture with consequent bleeding.

Moreover, the pathophysiology of SARS-CoV-2 infection is characterized by an increase of proinflammatory cytokines in serum (systemic cytokine storm), directly correlated with both disease severity and subtle coagulation disorders. Furthermore, it has been hypothesized that widespread endothelial cell damage occurs. Functional implications of this pathogenic mechanism include diffuse microvascular damage with both a substantial component of microvascular thrombosis (microCLOTS hypothesis) and imbalances in platelet recruitment. The latter could then result in multiple bleeding foci, typically affecting the distal microcirculation [5].

In the literature there are few data about spontaneous bleeding in COVID-19. On one hand, the prothrombotic features have been underlined and there are few reports about minor or occult and major internal bleeding observed in COVID-19 patients.

In one report, Shamkari et al. described bleeding and thrombotic manifestation in a cohort of 400 COVID-19 critically ill patients. In this study the authors found a very low rate of disseminated intravascoular coagulation $(2 \%$ of critically ill patients) [6].

In the cases we describe, no patient met the criteria for DIC according to the guidelines by the International Society for Thrombosis and Haemostasis [7]. D-dimer levels were moderately

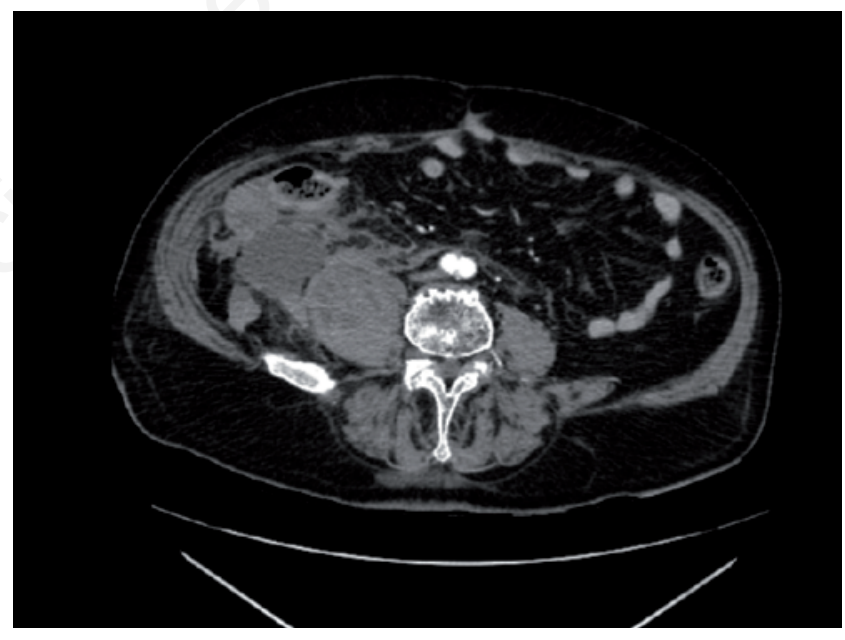

Figure 1. Case \#2. Abdominal CT scan of the patient \#2, showing large pelvic blood collection in the right iliac fossa.

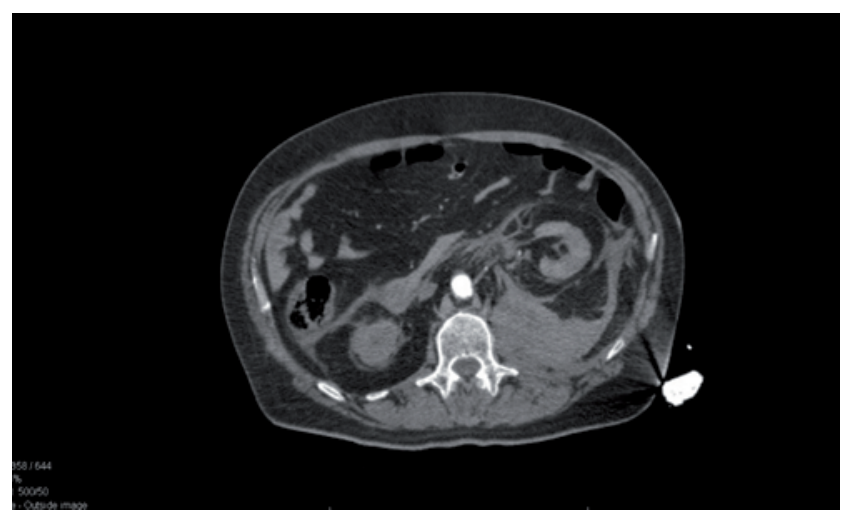

Figure 2. Case \#3. CT scan revealing large blood collection in the left ileopsoas muscle. 
increased and there were not any abnormalities in the PT/INR, activated PTT, fibrinogen level, or platelet count; these findings are uncharacteristic of DIC. Moreover, we know D-dimer levels are positively correlated with the prognosis of COVID -19 and the anticoagulant therapy may benefit COVID -19 patients, especially those without cardiovascular diseases [8].

In a report, Palumbo et al. observed that among the 818 consecutive patients with a positive nasopharyngeal swab test for SARS-CoV-2 admitted to the emergency department between March 1 and April 30, 2020, 16 (1.9\%) experienced at least one severe arterial bleeding episode [3]. In a letter to the editor, Conti et al described two cases of major bleeding in patients affected by severe SARS-CoV-2-related pneumonia without predisposing causes [10].

The cases we have described are emblematic because they describe episodes of major bleeding in patients with a low risk of bleeding. The clinical presentation was similar, with acute onset characterized by abdominal pain, severe acute anaemia and concomitant hypotension, but had different levels of gravity, different treatment and different prognosis. In the first case, an elastocompressive dressing and transfusion therapy were sufficient to normalize clinical parameters in few days. In the second case, the bleeding was more severe and embolization treatment of the right hypogastric artery was necessary to stop the bleeding and obtain resolution of the haemorrhage and normalization of clinical parameters. In the last case, the bleeding was very severe, and manifested as haemorrhagic shock, so much so that surgery was necessary, but the patient died in preparation for surgery.

These cases, underlining the importance of close monitoring of coagulation parameters and haemoglobin values in patients affected by severe COVID-19 and spontaneous iliopsoas haematomas should be considered in the differential diagnosis of abdominal pain in patients treated with LMWH even if at prophylactic dosage.

\section{References}

1. Richmann DD, Whitley RJ, Hayden FG, eds. Clinical virology, 4th ed. Washington: ASM Press; 2016.

2. Tang N, Li D, Wang X, et al. Abnormal coagulation parameters are associated with poor prognosis in patient with novel coronavirus pneumonia. J Thromb Haemost 2020;18:844-7.

3. Wang T, Chen R, Liu C, et al. Attention should be paid to venous thromboembolism prophylaxis in the management of COVID-19. Lancet Haematol 2020;7:e362-3.

4. Marquardt G, Barduzal Angles S, Leheta F, et al. Spontaneous haematoma of the iliac psoas muscle: a case report and review of the literature. Arch Orthop Trauma Surg 2002;122:109- 11.

5. Ciceri F, Beretta L, Scandroglio AM. Microvascular COVID19 lung vessels obstructive thromboinflammatory syndrome (MicroCLOTS): an atypical acute respiratory distress syndrome working hypothesis. Crit Care Resusc 2020;22:95-7.

6. Al-Samkari H, Karp Leaf RS, Dzik WH, et al. COVID-19 and coagulation: bleeding and thrombotic manifestations of SARSCoV-2 infection. Blood 2020;136:489-500.

7. Taylor FB Jr, Toh CH, Hoots WK, et al. Towards definition, clinical and laboratory criteria, and a scoring system for disseminated intravascular coagulation. Thromb Haemost 2001;86:1327-30.

8. Li Y, Zhao K, Wei H, et al. Dynamic relationship between Ddimer and COVID-19 severity. Br J Haematol 2020;190:e24-7.

9. Palumbo D, Guazzarotti G, De Cobelli F. Spontaneous major hemorrhage in COVID-19 patients: Another brick in the wall of SARS-CoV-2-associated coagulation disorders? J Vasc Interv Radiol 2020;31:1494-6.

10. Conti CB, Henchi S, Coppeta GP, et al. Bleeding in COVID19 severe pneumonia: The other side of abnormal coagulation pattern? Eur J Intern Med 2020;77:147-9. 